\title{
Pediatric severe pseudomembranous enteritis treated with fecal microbiota transplantation in a 13-month-old infant
}

\author{
JIAYI WANG ${ }^{1,2}$, YONGMEI XIAO ${ }^{1}, \mathrm{KAI} \mathrm{LIN}^{1}$, FEIFEI SONG ${ }^{1}$, TING GE ${ }^{1}$ and TING ZHANG ${ }^{1}$ \\ ${ }^{1}$ Department of Gastroenterology, Hepatology and Nutrition, Shanghai Children's Hospital; ${ }^{2}$ Department of Anesthesiology, \\ Shanghai Ninth People's Hospital, School of Medicine, Shanghai Jiao Tong University, Shanghai, P.R. China
}

Received September 15, 2014; Accepted October 20, 2014

DOI: $10.3892 /$ br.2014.403

\begin{abstract}
Fecal microbiota transplantation (FMT) is a procedure used to restore the intestinal microbiota of a diseased individual using indigenous intestinal microorganisms from a healthy donor. The current case report presents the first case of a 13-month-old male with severe pseudomembranous enteritis (PME) treated with FMT. The infant was admitted to Shanghai Children's Hospital with a 2-month history of diarrhea, and a 1.5-month history of retractable edema, hypoalbuminemia, electrolyte disturbance and malnutrition. Besides necessary nutritional support, the patient was treated twice with oral metronidazole combined with or without vancomycin. Diarrhea was partially remitted. However, the infant had bloody or dark-green feces, and a distended abdomen. On day 96 from the initiation of the disease, a single FMT via a nasal jejuna feeding tube was performed. From day 2 until 4 months post-FMT, the patient presented with no diarrhea, normal feces and a satisfactory weight. To the best of our knowledge, this is the first pediatric PME treated with FMT. The current data show that FMT is an efficient choice for recurrent clostridium difficile infection and PME in adults and a few pediatric cases. Due to a lack of safety and effectiveness data, treatment should be cautiously applied in the pediatric population.
\end{abstract}

\section{Introduction}

Pseudomembranous enteritis (PME) is an acute inflammatory bowel disease affecting the total population. Clostridium difficile $(C$. difficile) is recognized as one of the most important pathogens responsible for antibiotic-associated colitis and accounts for $15-25 \%$ of nosocomial antibiotic-associated

Correspondence to: Dr Ting Zhang, Department of Gastroenterology, Hepatology and Nutrition, Shanghai Children's Hospital, School of Medicine, Shanghai Jiao Tong University, 355 Luding Road, Putuo, Shanghai 200062, P.R. China

E-mail: zhangting_shmu@163.com

Key words: pseudomembranous enteritis, fecal microbiota transplantation, children, clostridium difficile infection diarrhea cases (1). Up to $70 \%$ of infants may be asymptomatically colonized with $C$. difficile, including toxigenic strains. The rates of colonization decrease with age, falling to $6 \%$ by 2 years of age. The colonization rates in children over the age of 2 years are similar to those in adults $(\sim 3 \%)(2)$. Currently, the standard treatment recommendations for mild to moderate $C$. difficile infection (CDI) include metronidazole or vancomycin (1). However, the cure rates of severe CDI treated with metronidazole and vancomycin are only $76 \%$ and $97 \%$, respectively. The chance of recurrent CDI within 8 weeks is $10-20 \%$, but once a patient experiences recurrence, the rate of further recurrences increases to $40-65 \%$ (3). Given the effective outcome, low cost, apparent safety and availability of fecal microbiota transplantation (FMT), it has become an increasingly accepted option for treatment of CDI (4). Microbiota dysbiosis is the fundamental pathogenetic base of PME/CDI/antibiotic-associated diarrhea, and FMT provides a rapid solution to restore the intestinal microbiota (5). To the best of our knowledge, the current study presents the first case of a 13-month-old infant with severe PME who was treated with FMT in China $(6,7)$.

\section{Case report}

A 13-month-old male was admitted to the Shanghai Children's Hospital (Shanghai, China) with a 2-month history of diarrhea, loose, malodorous dark-green stools with variable mucus and blood, and a 1.5-month history of retractable edema, hypoalbuminemia, electrolyte disturbance and severe malnutrition. Initially, the infant presented with acute diarrhea for 1 day, followed by ileus and exploratory laparotomy and high ligation of inguinal hernia on day 3 without a noticeable food allergy, family history and vaccination. Multiple broad-spectrum antibiotics, including ceftazidime, amoxicillin, imipenem, metronidazole and vancomycin, were introduced daily to the patient during 2 months. The patient had a birth weight of 2,500 $\mathrm{g}$ with a 20-day history of diarrhea at the age of six months.

Physical examination revealed that the infant was conscious, weak and responding. The vital signs were stable, the weight was $8.4 \mathrm{~kg}$ and the eyelids were puffy and had dry skin and mucous. Abdominal examinations showed abdominal distention, pitting edema of the pretibial area and the dorsum, and normal neurological examination. The dark-green stools were 


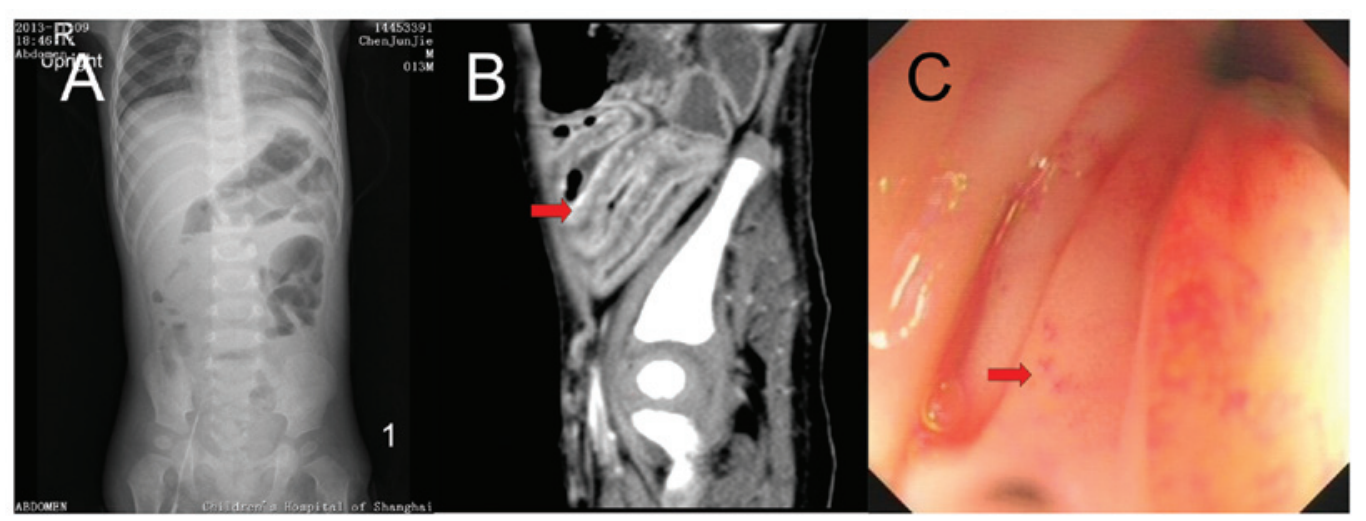

Figure 1. (A) X-ray showing a dilated bowel. (B) Enhanced abdominal computed tomography revealing a swallowed-segmented intestinal wall (red arrow). (C) Colonoscopy revealing left colitis with erosion, erythematous and edema (red arrow).

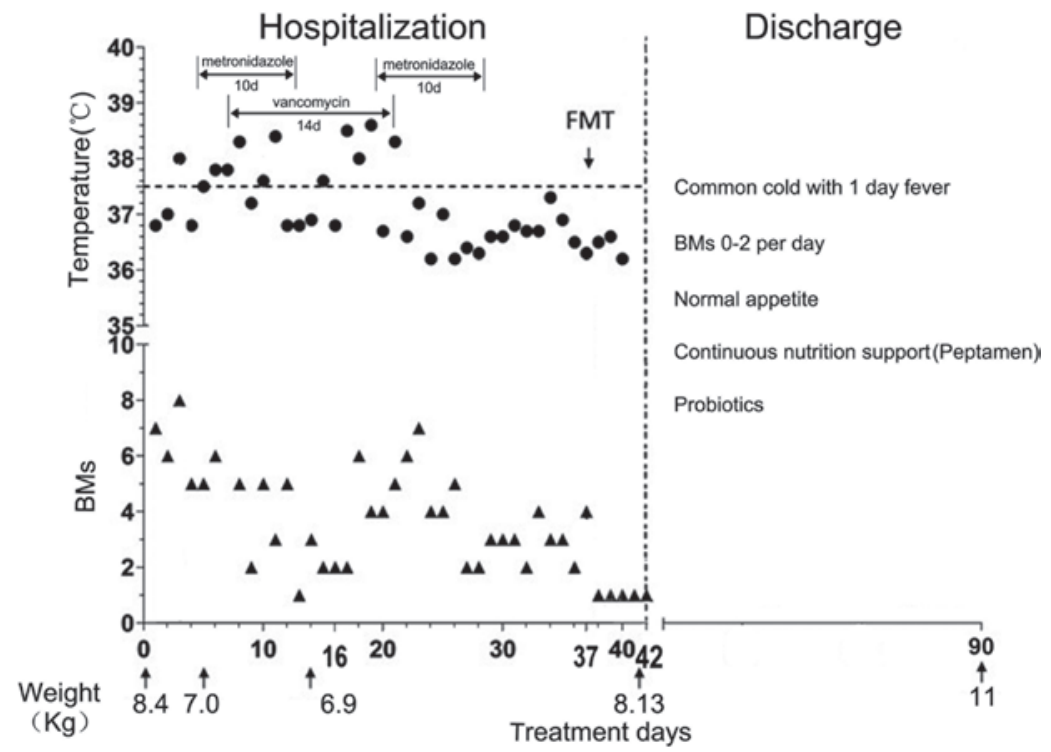

Figure 2. Temperature, stool frequency, weight, major antibiotics and medical procedure patterns. BM, bowel movements (stool frequency); d, days.

loose, malodorous and had a frequency of 5-8 times per day with variable mucus and positive occult blood.

The routine blood examination showed white blood cells (WBC), 10.7-30.3 x109/1; hemoglobin, 85-129 g/l; platelet count, 305-616 x10 $/ 1$; and C-reactive protein, 3-19 mg/1. The blood biochemical examination demonstrated albumin, 11-38 g/l; globulin, 10-16 g/l; $\mathrm{K}^{+}, 2.8-5.5 \mathrm{mmol} / \mathrm{l} ; \mathrm{Na}^{+}$, 131-143 mmol/1; and $\mathrm{Ca}^{2+}, 1.69-2.25 \mathrm{mmol} / 1$. The routine stool examination showed WBC, 2-20/high power (HP); red blood cells, 0-15/HP; and positive fecal occult blood test. Multiple stool cultivation was negative. The fecal bacteria population test showed $50 \%$ enterobacteria and $50 \%$ enterococcus on admission. Fecal $C$. difficile toxin $\mathrm{A}$ and $\mathrm{B}$ was negative during days 11-13 of hospitalization. Other laboratory tests showed no significant abnormalities. Abdominal images, including ultrasound, X-ray and enhanced computed tomography revealed a swallowed-segmented intestinal wall, cellulitis, ascites and pleural effusion. Colonoscopy on day 16 of hospitalization showed left colitis (Fig. 1).

The condition of the infant was improved with nutritional support, correction of electrolytes, acid-base disturbance, supply of albumin, and intravenous immunoglobulin. Edema gradually faded on day 7 and completely disappeared on day 14 following hospitalization. The patient was treated with two rounds of oral metronidazole for 10 days, with vancomycin for 14 days, and finally a single FMT (the feces donor was the mother of the patient) was performed via a nasal jejunal feeding tube on day 96 following the initiation of the disease, which was day 37 from admission. The patient completely recovered and was released on day 5 post-FMT. The temperature, stool frequency, weight, major antibiotics and medical procedure patterns are shown in Fig. 2. Normal appetite, normal bowel movement frequency and satisfactory weight were exhibited during the 4 months post-FMT.

\section{Discussion}

Based on the clinical features and the response of whole treatment, severe PME was the final diagnosis of the infant in the present study. The diagnosis of PME was based on the following reasons: i) Diarrhea was persistent, and there were intestinal mucosae in the stools more than once; ii) the patient presented risk factors, including multiple broad-spectrum antibiotics application, long-term fasting, exploratory laparotomy and high 
ligation of inguinal hernia; and iii) although the use of metronidazole and vancomycin in the Children's Hospital (Jiangxi, China) was ineffective, empirical treatment with adequate dosage and duration of metronidazole combined with vancomycin showed partial effectiveness in Shanghai Children's Hospital (Shanghai, China). The effectiveness of FMT was positive.

According to the guidelines by the Society for Healthcare Epidemiology of America (SHEA) and the Infectious Diseases Society of America (IDSA) (1), the definition for the usual presentation of CDI includes the following findings: i) The presence of diarrhea, defined as passage of $\geq 3$ unformed stools in $\leq 24$ consecutive hours; and ii) a stool test result positive for the presence of toxigenic $C$. difficile or its toxins, or colonoscopic or histopathological findings demonstrating pseudomembranous colitis. The guideline also indicated the difficulties for CDI diagnosis. SHEA-IDSA defined severe CDI as leukocytosis with a WBC of $\geq 15,000 / 1$ or a serum creatinine level $\geq 1.5$ times the premorbid level (1). The European Society of Clinical Microbiology and Infectious Diseases defined severe CDI as serum albumin $<3 \mathrm{~g} / \mathrm{dl}$ plus one of the following; WBC $>15 \times 10^{9} / 1$ and abdominal tenderness (3). Although, $C$. difficile toxin tests during the hospitalization were all negative, possibly due to the antibiotic treatment prior to the toxin tests. Colonscopy was not performed until the infant reached a stable general condition (day 16 of hospitalization). Relatively late timing may be the reason for missing the typical image findings and possible positive $C$. difficile detection.

In the present case, diarrhea was persistent, serum albumin was $<1 \mathrm{~g} / \mathrm{dl}$, and WBC was $>15 \times 10^{9} / 1$ for many examinations, with the highest being 30x10 $/ 1$. Abdominal images revealed swallowed-segmented intestinal sigmoid colon and rectum. The temperature of the patient was $>38.5^{\circ} \mathrm{C}$. All the parameters of the present case assisted in the diagnosis of severe CDI. In China, there are only a few case reports regarding severe CDI (8-11). None of the studies are supported by the toxin, fecal bacteria population test and culture. Due to the population size of China, antibiotic abuse and the few cases reported, severe CDI is extremely under-recognized, particularly in children. In the next few years, more progress is required for pediatric CDI in China, including clinical diagnosis, treatment and research.

\section{References}

1. Cohen SH, Gerding DN, Johnson S, Kelly CP, Loo VG, McDonald LC, et al: Clinical practice guidelines for Clostridium difficile infection in adults: 2010 update by the society for healthcare epidemiology of America (SHEA) and the infectious diseases society of America (IDSA). Infect Control Hosp Epidemiol 31: 431-455, 2010

2. Sammons JS, Toltzis P and Zaoutis TE: Clostridium difficile Infection in children. JAMA Pediatr 167: 567-573, 2013.

3. Surawicz CM, Brandt LJ, Binion DG, Ananthakrishnan AN, Curry SR, Gilligan PH, et al: Guidelines for diagnosis, treatment, and prevention of Clostridium difficile infections. Am J Gastroenterol 108: 478-498, 2013.

4. Brandt LJ, Aroniadis OC, Mellow M, Kanatzar A, Kelly C, Park T, et al: Long-term follow-up of colonoscopic fecal microbiota transplant for recurrent Clostridium difficile infection. Am J Gastroenterol 107: 1079-1087, 2012.

5. Borody TJ, Warren EF, Leis SM, Surace R, Ashman O and Siarakas S: Bacteriotherapy using fecal flora: toying with human motions. J Clin Gastroenterol 38: 475-483, 2004.

6. Russell G, Kaplan J, Ferraro M and Michelow IC: Fecal bacteriotherapy for relapsing Clostridium difficile infection in a child: a proposed treatment protocol. Pediatrics 126: e239-242, 2010.

7. van Nood E1, Vrieze A, Nieuwdorp M, Fuentes S, et al: Duodenal infusion of donor feces for recurrent Clostridium difficile. $\mathrm{N}$ Engl J Med 368: 407-415, 2013.

8. He JQ, Li JD and Mo QH: Severe pseudomembranous enterocolitis-one case report and review of literature. Hainan Med J 19: 137-138, 2008 (In Chinese)

9. Li WH, Liu XL and Tian YL: Clinical analysis of 62 psendomembranons colitis patient. Chongqing Med J 41: 2379-2381, 2001 (In Chinese).

10. Wang L, Gao J and Cheng WP: Clinical analysis of 9 psendomembranons colitis patient. Pract Clin Med 13: 22-30, 2012 (In Chinese).

11. Zhang L, Zhang YD, Duan LF, Liang Y and Gong QM: Severe pseudomembranous colitic induced by antibiotics in 1 patient. Chin J Clin Pharmacol 26: 684-686, 2010 (In Chinese). 